Egyptian

Orthodontic Journal

\title{
The effects of utilizing conventional etching versus self etch primer on the enamel color change after treatment with fixed orthodontic appliances. In vivo study
}

\author{
Ahmad Mohammad Hafez*
}

ABSTRACT:

Objectives: The present study was conducted to evaluate clinically the effect of conventional etching and the self etch primer on the change in enamel color before and after orthodontic treatment. Material and Methods: The study was conducted on 24 patients treated with fixed orthodontic appliances. Metal brackets were bonded to the teeth mesial to first molars. The brackets were bonded utilizing Transbond XT adhesive after application of either conventionaletching (\%37 phosphoric acid etch and Transbond XT primer) or Transbond XT self etch primer (split mouth design). Teeth which showed bracket bond failure were excluded from the study. Therefore, the final number of teeth which were included in the conventional etch and self etch groups were 164 and 160 respectively. The color of the labial and buccal surfaces of the teeth mesial to the first molars was evaluated before bracket bonding and after orthodontic treatment. This step was done with the aid of spectrophotometer Vita Easyshade (Nita Zahnfabri, Germany). The evaluation of the color depended on the color space system of CIELAB (Commission Internationale de C.Eclairage $\mathcal{L}^{\star}$, $a^{\star}$ and $\left.6^{\star}\right)$. Three parameters were measured $\left(\mathcal{L}^{\star}, a^{\star}, 6^{\star}\right)$. Also, color change (IE) was calculated. Then the collected data was statistically analyzed using Paired $t$-test.

Results: All color measurements $\left(\mathcal{L}^{\star}, a^{\star}, b^{\star}\right)$ were significantlydecreasedafterorthodontictreatment $(\mathbb{P}<0.05)$. Conventional etching provided significantly higher $\triangle E$ v value than the self etch primer $(\mathbb{P}<0.05)$. Conclusion: The utilization of conventional etching or selfetch primer have a significant negative effect on tooth color after orthodontic treatment. However, the conventional etching has higher pronounced effects.

\section{INTRODUCTION}

Bonding of orthodontic brackets to tooth enamel is considered one of the basic procedures in orthodontic practice. The popularity of this technique over banding gained from its simplicity, better esthetics, less gingival irritation and elimination of post treatment band spaces closure. $^{1,2}$

The adhesive system considered one of the most important factors that affect the success of such technique. The ideal adhesive system should provide sufficient bond strength to withstand orthodontic and masticatory forces without any negative effects on the tooth structure. ${ }^{3-7}$ In line with this demand, Primers and adhesives of different composition and curing modes have been developed aiming to enhance the bond strength and reduce the failure rates of the brackets. The traditional adhesive system consists

\footnotetext{
* Lecturer, Department of Orthodontics, Faculty of Dentistry, Mansoura University, Mansoura, Egypt
} 
of etchant, primer, and adhesive resin. The etchant dissolves the enamel crystals in the prism structure leading to microscopic undercuts and rough surfaces that provide micromechanical retention via the formation of resin tags. Later on, the self-etching primer (SEP) was introduced combining the etchant and primer in a single solution aiming to reduce the bonding steps, time, and exposure to saliva. However, SEP has lower etching ability than the traditional total etch system. Therefore it reduces the enamel damages potential..$^{8-13}$

The orthodontist and patients satisfaction to the orthodontic treatment outcomes could be negatively affected by the changes in enamel color or quality. Tooth color could be affected by aging, dietary, medication, and dental materials such as adhesives and restorations. ${ }^{14}$

Several studies have been done to evaluate the effect of orthodontic treatment on the tooth color. However the results showed a large degree of controversy. Karamouzos et al, Al Maaitah et al and Corekci et al evaluated the tooth color before and after treatment and reported that orthodontic treatment has a pronounced effect on the tooth color. ${ }^{15-17}$ Eliades et al assessed the color stability of chemically and light cured orthodontic adhesive resins subjected to artificial photoageing. ${ }^{18}$ The results revealed that both adhesives exhibited color change. Faltermeier et al studied the effect food dyes and ultraviolet light on discoloration of orthodontic adhesives. ${ }^{19}$ The tested adhesives had unsatisfactory color stability that could affect the tooth color. Boncuk et al reported that orthodontic treatment alters the original color of enamel, and both the adhesive system and the resin-removal methods are responsible for this change. ${ }^{20}$ Joo et al compared the influence of conventional acid etching and self etch primer on the stain susceptibility of enamel surface after debonding. ${ }^{21}$ They found that SEP showed less stain susceptibility if the thin residual adhesive layer is removed by polishing. Trakyali et al evaluated the enamel color changes before bracket bonding, after debonding, and removal of excess adhesives, and after polishing. ${ }^{22}$ Their results revealed that color changes induced by photoageing cannot be clinically observed. Jahanbin et al studied the effect of adhesive types on enamel discoloration around orthodontic brackets. ${ }^{23}$ According to their findings the type of adhesive had no effect on enamel color change. Later on, Chen et al concluded that there is no strong evidence that the orthodontic treatment with fixed appliances alters the original color of the enamel. ${ }^{24}$

The present study was conducted to evaluate clinically the effect of conventional etching and the self etch primer on the change in enamel color before and after orthodontic treatment.

\section{Materials and methods}

The study was conducted on 24 patients (12 females and 12 males) seeking orthodontic. The sample size was estimated by $\mathrm{G}^{*}$ Power software (version 3.0.10). Assuming type I statistical error of $5 \%$ and 2-tailed statistical test, this study was designed to have a power of $90 \%$ based on the previous study evaluated the effect of different etching techniques on tooth color. ${ }^{16}$ The mean Commission Internationale de l'Eclairage values for all studied teeth before and after orthodontic treatment were $0.10+$ 1.51 and $0.42+-1.50$ respectively. Therefore, the calculated sample size was 235 teeth. Since, we had a split mouth trial design with 1:1 allocation ratio, ${ }^{15}$ patients (118 teeth per trial arm) were required for this study. To guard against dropouts, the sample was increased to ${ }^{24}$ patients.

The patients were selected according the 
following criteria; age range from ${ }^{18-22}$ year and the teeth had intact enamel surface, free from any pits, cracks, caries, dental fluorosis or other hypomineralized lesions or pigmentation. The maxillary and mandibular first premolars were extracted as a part of the orthodontic treatment in all patients. Metal brackets Roth prescription and 0.022 inch slot (Ormoco, USA) were bonded to the teeth mesial to first molars. In half of the patients, in the right maxillary and left mandibular quadrants conventional etching was utilized, while self etch primer was used in the left maxillary and right mandibular quadrants. In the other half of the patients the revers was done (split mouth design). Teeth which showed bracket bond failure were excluded from the study. Therefore, the final number of teeth which were included in the total etch and self etch groups were 164 and 160 respectively (Table 1).

The bonding procedures were done according to the manufactures instructions. The labial and buccal surfaces of the teeth were polished with non fluoridated pumice and rubber cup. Then, in half of the teeth, 37\% phosphoric acid (Eco-Etch, Ivoclar Vivadent, Liechtenstein) was applied to the enamel surfaces for 30 seconds (conventional etch). Then the teeth were washed thoroughly with water and dried with air. Thin coat of Transbond XT primer (3M, Unitek, Calif, USA) was applied to the etched dry enamel. Transbond XT adhesive resin (3M, USA) was applied to the bracket base. Finally, the bracket was placed in its correct position on tooth surface and pressed firmly. The excess bonding resin was removed using a sharp scaler. Then the adhesive was light cured for 20 seconds. On the other half of the teeth, all previous steps were followed except that Transbond XT self etch primer (3M, USA) was applied to the enamel surfaces for 5 seconds instead of the acid etchant and Transbond XT primer.

The same instructions for oral hygiene care and appliance maintenance were given for all the patients though out the treatment periods (22-25 month). After finishing the orthodontic treatment brackets were debonded using debonding plier. The excess adhesive was removed with carbide bur and enamel surface was smoothed by Sof- Lex finishing discs (3M, USA). This step was done with the aid of $4 \mathrm{X}$ dental eye loups (UNIVET, Italy).

\section{Measurement of the color change:}

The color of the labial and buccal surfaces of the teeth mesial to the first molars was evaluated before treatment (bracket bonding) and after treatment (debonding and removal of excess adhesives with carbide bur). This step was done with the aid of spectrophotometer Vita Easyshade (Vita Zahnfabrik, Bad Säckingen, Germany). This device has a digital pointer, 19 fiber optics for precise illumination of the tooth surface and spectrophotometric sensors for determination of color in numeric way. The tip of the spectrophotometer was positioned perpendicular and flushed to the center of labial or buccal surfaces of the crowns of the teeth $1 \mathrm{~mm}$ from the incisal edges or cusp tips. Three shade readings were taken for each tooth. Calibration of the spectrophotometer was done every time before each color measurement. All measurements were taken under the same dental unit lamp and the teeth were wet not dry.

The evaluation of the color depends on the color space system of CIELAB (Commission Internationale de l'Eclairage $\mathrm{L}^{*}$, $\mathrm{a}^{*}$ and $\left.\mathrm{b}^{*}\right)$. This system utilizing 3 parameters of color: degree or value of lightness $\left(\mathrm{L}^{*}\right)$ which started from 0 that means black and ended by 100 which means white, measurement a ranging from positive a* which equal to red to negative $a^{*}$ which equal to green, and measurement $b^{*}$ where positive $b^{*}$ means yellow and negative $\mathrm{b}$ that means blue. Finally color change $(\Delta \mathrm{E})$ was calculated by the following equation:- 
$\Delta \mathrm{E}=\left[\left(\mathrm{L}^{*}{ }_{2}-\mathrm{L} *{ }_{1}\right) 2+\left(\mathrm{a}_{2}^{*}{ }_{2}-\mathrm{a}^{*}{ }_{1}\right) 2+\left(\mathrm{b}_{2}{ }_{2}-\mathrm{b}^{*}{ }_{1}\right)^{2}\right]^{1 / 2.15}$

\section{Statistical analysis:}

The collected data was statistically analyzed using SPSS Statistics for Windows, version 20.0. (IBM Corp, USA). The data was expressed as mean and standard deviation (SD). Paired t-test was used to determine the significant differences between the pre and postreatment $\mathrm{L}^{*}, \mathrm{a}^{*}$ and $\mathrm{b}^{*}$ color measurements for each adhesive system and between $\Delta \mathrm{E}$ of the two adhesive systems. All tests were conducted at 0.05 level of significance.

\section{Results}

The means and standard deviations of the color measurements $\left(\mathrm{L}^{*}, \mathrm{a}^{*}, \mathrm{~b}^{*}\right)$ of the conventional etch and self etch groups and the results of t test are presented in tables 2-4. All before bracket bonding color measurements were significantly higher than those after orthodontic treatment $(\mathrm{P}<0.05)$. The means and standard deviations of the $\Delta \mathrm{E}$ measurements of the conventional etch and self etch groups and the results of $t$ test are presented in table 5 . The $\Delta \mathrm{E}$ of the conventional etch group was significantly higher than that of self etch group $(\mathrm{P}<0.05)$

Table 1: The number of teeth in the two studied groups.

\begin{tabular}{|l|c|c|c|}
\hline \multicolumn{1}{|c|}{ Group } & Total teeth number & $\begin{array}{c}\text { Teeth with bracket } \\
\text { bond failure }\end{array}$ & $\begin{array}{c}\text { Teeth without bracket } \\
\text { bond failure }\end{array}$ \\
\hline Convential etch & 176 & 12 & 164 \\
\hline Self etch & 176 & 16 & 160 \\
\hline
\end{tabular}

Table (2): The Mean and standard deviation (SD) of color measurement $\mathrm{L}^{*}$ before bracket bonding and after debonding and removal of excess adhesive and the results of paired t-test.

\begin{tabular}{|c|c|c|c|c|}
\hline \multirow{2}{*}{ Group } & \multicolumn{2}{|c|}{ Mean \pm SD } & \multirow{2}{*}{$\mathrm{t}$} & \multirow{2}{*}{$\mathrm{P}$} \\
\hline & Before bonding & After debonding & & \\
\hline Conventional etch & $90,78 \quad \pm 3,06$ & $\begin{array}{ll}84,06 & \pm 3,27\end{array}$ & 34,705 & 0,000 \\
\hline Self etch & $90,52 \quad \pm 3,09$ & $85,27 \quad \pm 3.15$ & 39,013 & 0,000 \\
\hline
\end{tabular}

Significance: $\mathrm{P} \leq 0.05$.

Table (3): The Mean and standard deviation (SD) of color measurement a* before bracket bonding and after debonding and removal of excess adhesive and the results of paired sample t-test.

\begin{tabular}{|l|c|c|c|c|}
\hline \multirow{2}{*}{ Group } & \multicolumn{2}{|c|}{ Mean \pm SD } & \multirow{2}{*}{$\mathrm{t}$} & \multirow{2}{*}{$\mathrm{P}$} \\
\cline { 2 - 3 } & Before bonding & After debonding & & 0,000 \\
\hline Conventional etch & $1,69 \quad \pm 0,25$ & $0,85 \quad \pm 0,23$ & 69,404 & 0,000 \\
\hline Self etch & $1,68 \quad \pm 0,25$ & $0,89 \quad \pm 0,25$ & 70,012 & \\
\hline
\end{tabular}

Significance: $\mathrm{P} \leq 0.05$. 
Table (4): The Mean and standard deviation (SD) of color measurement $b^{*}$ before bracket bonding and after deponding and removal of excess adhesive and the results of paired sample t-test.

\begin{tabular}{|c|c|c|c|c|}
\hline \multirow{2}{*}{ Group } & \multicolumn{2}{|c|}{ Mean \pm SD } & \multirow{2}{*}{$\mathrm{t}$} & \multirow{2}{*}{$\mathrm{P}$} \\
\hline & Before bonding & After deponding & & \\
\hline Conventional etch & $29,79 \quad \pm 2,96$ & $26,06 \quad \pm 2,96$ & 63,431 & 0,000 \\
\hline Self etch & $30,10 \quad \pm 2,85$ & $25,95 \quad \pm 2,86$ & 60,611 & 0,001 \\
\hline
\end{tabular}

Significance: $\mathrm{P} \leq 0.05$.

Table (5): The mean and standard deviation (SD) of $\Delta \mathrm{E}$ of the two studied groups and the results of paired sample t-test.

\begin{tabular}{|l|c|c|c|}
\hline \multicolumn{1}{|c|}{ Group } & Mean $\pm \mathrm{SD}$ & $\mathrm{t}$ & $\mathrm{P}$ \\
\hline Conventional etch & $7,89 \pm 2,07$ & \multirow{2}{*}{7,725} & 0,000 \\
\hline Self etch & $6,86 \pm 1,42$ & \\
\hline
\end{tabular}

Significance: $\mathrm{P} \leq 0.05$.

\section{Discussion}

The present study was conducted to evaluate clinically the effect of bonding orthodontic brackets with adhesive systems including either conventional etching or self etch primer on the change in enamel color before and after orthodontic treatment. Bracket bonding and debonding, removal of excess adhesive and finishing and color assessment were done by the same technique and in the same environment. Different resin removal techniques could affect the enamel color with different degree as it cause enamel roughness. Technique that provides smoother enamel surface will lead to increase light reflection and hence lesser color change occur. ${ }^{20}$ Dental eye loups was utilized to avoid unnecessary enamel abrasion during removal of excess adhesive that could affect tooth color.

Spectrophotometer was used for color assessment. It considered one of the most accurate devices for tooth color evaluation. The accuracy of this device was investigated in several studies. Color readings were taken depending on Munsell's percentile units color system. This system produces numeric information with high correlation to the actual visual response. ${ }^{25,26}$ Color measurements were done under the same surrounding light sours (same room and light of the dental unit) to avoid any effect of different light sources on the color measurements that could lead to bias in the results. Also, the teeth were kept wet during measurements to avoid changes from dryness on the color.

The results of the present study revealed that the $L^{*}, a^{*}, b^{*}$ measurements were significantly decreased after orthodontic treatment $(\mathrm{P}<0.05)$. It was reported that changes in $\mathrm{L}^{*}$ measurements can be visually detected easily and preciously more than measurements $a$ and $b .^{22}$ The change in $L^{*}$ measurements was more than 5 units. According to Paul et al, change in $\mathrm{L}^{*}$ more than 2 unites will lead to visible color changes. ${ }^{27} \mathrm{In}$ addition, $\Delta \mathrm{E}$ in the present study was 7.7 and 5.4 for total etch and self etch 
primer respectively. These values exceed the clinically accepted once. $\Delta \mathrm{E} 3.7$ more is considered clinically not acceptable as it leads to pronounced tooth color change that could be detected by human eye. ${ }^{22,28}$ The results also revealed that there was significant difference between $\Delta \mathrm{E}$ of either etchant system $(\mathrm{P}<0.05)$. These previous findings could be explained by the penetration of the resin tags into the enamel surface that cannot be completely removed by debonding and finishing procedures. These resin tags could absorb food colorants and products arising from the corrosion of the orthodontic appliance leading too enamel discoloration. In addition, the enamel surface become rougher after bracket debonding and enamel finishing. This will change the refractive index and subsequently the reflected light component. ${ }^{17,20}$ Another important factor that could lead to tooth color change is the development of enamel decalcification. This change in the enamel mineral is accompanied by increasing its porosity and change in its optical characteristics leading to the appearance of white discoloration. In addition the significant differences between $\Delta \mathrm{E}$ of the two adhesive systems could be attributed to the conventional etch has higher etching effects than the self etch one and hence deeper resin tags were developed with the conventional etch. ${ }^{29}$

The findings of the present study were in harmony with those of Eliades et al and Boncuk et al. ${ }^{18,20}$ On the other hand, they were in disagreement with those of Karamouzos et al, ${ }^{15}$ and Corekci et $\mathrm{al}^{17}$ and Trakyali et al,22 who reported $\Delta \mathrm{E}$ less than 3.7 while in the present study it was $7.89 \pm 2.07$ and $6.86 \pm 1.42$ for conventional etch and self etch groups respectively. In addition, Al Maaitah et al found no significant differences between either conventional etch or self etch. ${ }^{16}$ This controversy could be attributed to the differences in the adhesive system used, sample size or the study period. The longer the study period the longer the presence of resin tag in the enamel tags with increase the potential of tooth discoloration. In addition the risk of enamel decalcification occurrence increase.

\section{Conclusions}

The utilization of conventional etching or self etch primer have a significant negative effect in tooth color after orthodontic treatment. However, the conventional etching has higher pronounced effects.

\section{References}

1. Bishara SE, Oonsombat C, Soliman MMA, Warren JJ, Laffoon JF, Ajlouni R. Comparison of Bonding Time and Shear Bond Strength Between a Conventional and a New Integrated Bonding System. Angle Orthod 2005;75:237-242.

2. Talpur M, Cunningham SJ, Moles DR, Steven P. Jones SP. The relationship between base dimensions, force to failure, and shear bond strengths of bondable molar tubes. Angle Orthod 2012; 82: 536-540.

3. Faltermeier A, Behr M, Müssigc D. A comparative evaluation of bracket bonding with 1-, 2-, and 3-component adhesive systems. Am J Orthod Dentofacial Orthop 2007;

132:144.e1-144.e5.

4. Urabe H, Rossouw PE, Titley KC, Yamin C. Combination of etchants, composite resins, and bracket systems: An important choice of orthodontic bonding procedures. Angle Orthod 1999;69:267-275.

5. Soderquist SA, Drummond JL, Evans CA. Bond strength evaluation of ceramic and stainless steel bracket bases subjected to cyclic tensile loading. Am J Orthod Dentofacial Orthop. 2006;129:175.e7-175.e12. 
6. Daub J, Berzins DW, Linn BJ, Bradley TG. Bond strength of direct and indirect bonded brackets after thermocycling. Angle Orthod 2006;76:295-300.

7. Reynolds IR. A review of direct orthodontic bonding. Br J Orthod, 1979: 2: 171-178.

8. Davari AR, Yassaei S, Daneshkazemi AR, Yosefi MH. Effect of different types of enamel conditioners on the bond strength of orthodontic brackets. J Contemp Dent Pract 2007;8:36-43.

9. Ozer M, Bayram M, Dincyurek C, Tokalak F. Clinical bond failure rates of adhesive precoated self-ligating brackets using a self-etching primer. Angle Orthod. 2014: Vol. 84:155-160.

10. Vicente A, Bravo LA, Romero M, Ortiz AJ, Canteras M. A Comparison of the shear bond strength of a resin cement and two orthodontic resin adhesive systems. Angle Orthod 2004;75:109-113.

11. Bishara SE, VonWald L, Laffoon JF, Warren J. Effect of using a new cyanoacrylate adhesive on the shear bond strength of orthodontic brackets. Angle Orthod, 2001; 71: 466-469.

12. Bishara SE, Ostby AM, Laffoon JF, Warren JJ. The effect of modifying the self-etchant bonding protocol on the shear bond strength of orthodontic brackets. Angle Orthod 2007; 77:504-508.

13. Cehreli ZC, Kecik D, Kocadereli I. Effect of self-etching primer and adhesive formulations on the shear bond strength of orthodontic brackets. Am J Orthod Dentofacial Orthop 2005;127:573-579.

14. Kitasako Y, Sadr A, Shimada Y, Sumi Y. The utility of chewing gum in treating white spot lesions. Curr Oral Health Rep. 2016;3:111-116.

15. Karamouzos A, Athanasiou AE, Papadopoulos MA, Kolokithas G. Tooth-color assessment after orthodontic treatment: a prospective clinical trial. Am J Orthod Dentofacial Orthop 2010;138:537.e1-537.e8.

16. Al Maaitah EF, Abu Omar AA, Al-Khateeb SN. Effect of fixed orthodontic appliances bonded with different etching techniques on tooth color: A prospective clinical study. Am J Orthod Dentofacial Orthop. 2013;144:43-49.

17. Corekci B, Toy E, Ozturk F, Malkoc S, Ozturk B. Effects of contemporary orthodontic composites on tooth color following short term orthodontic treatment: a controlled clinical study. Turk J Med Sci. 2015; 45:1421-1428.

18. Eliades T, Gioka C, Heim M, Eliades G, Makou M. Color stability of orthodontic adhesive resins. Angle Orthod. 2004; 74:391-393.

19. Faltermeier A, Rosentritt M, Reicheneder C, Behr M. Discoloration of orthodontic adhesives caused by food dyes and ultraviolet light. Eur J Orthod. 2008;30:89-93.

20. Boncuk Y, Cehreli ZC, Polat-Ozsoy O. Effects of different orthodontic adhesives and resin removal techniques on enamel color alteration. Angle Orthod. 2014;84:634-641.

21. Joo HJ, Lee YK, Lee GY, Kim TJ, Lim YK. Influence of orthodontic adhesives and clean-up procedures on the stain susceptibility of enamel after debonding. Angle Orthod 2011; 81:334-340. 
22. Trakyalı G, Ozdemir FI, Arun T. Enamel colour changes at debonding and after finishing procedures using five different adhesives. Eur J Orthod. 2009;31:397-401.

23. Jahanbin A, Ameri H, Khaleghimoghaddam R. Effects of adhesive types on enamel discolouration around orthodontic brackets. Aust Orthod J. 2009;25:19-23.

24. Chen Q, Zheng X, Chen W, Ni Z, Zhou Y. Influence of orthodontic treatment with fixed appliances on enamel color: a systematic review. BMC Oral Helth. 2015; 10:15-31.

25. Gustavo MS, Oliveira, Ritter AV, Heymann $\mathrm{HO}$ et al. Remineralization effect of CPP-ACP and fluoride for white spot lesions in vitro. $\mathrm{J}$ Dent. 2014;42:1592-1602.
26. Silame FDJ, Tonani R, Roman CCA, Chinelatti $\mathrm{M}$ et al. Color stability of temporary restorations with different thickness submitted to artificial accelerated aging. Eur J Prostbodont Rest Dent. 2013;21:187-190.

27. Paul S, Peter A, Pietroban N, Hammerle CHF. Visual and spectrophotometric shade analysis of human teeth. J Dent Res 2002;81:578-582.

28. Johnston WM, Kao EC. Assessment of appearance match by visual observation and clinical colorimetry. J Dent Res 1989;68:819-822.

29. Holzmeier M, Schaubmayr M, Dasch W, Hirschfelder U. A new generation of self-etching adhesives: comparison with traditional acid etch technique. J Orofac Orthop 2008;69:78-93. 